\title{
Scanning Electron Microscopes with Integrated Raman Spectrometer Revealing New Complementary Information
}

\author{
Stefanie Freitag ${ }^{1}$ \\ ${ }^{1}$ Carl Zeiss Microscopy GmbH, Market Segment Material Sciences, Munich Site, Germany
}

Scanning electron microscopes (SEM) delivers a magnified view of tiny structures in materials. The resolution goes down to a few nanometer. Different detectors like secondary electron detectors and back scattered detectors enable the investigation of samples in surface sensitive or contrast enhanced modes. In addition energy dispersive x-ray detectors (EDX) reveal information about the existing elements and metals in the specimen. This chemical analysis is limited to a certain atomic number (atomic number $Z=4$, Beryllium). To overcome this limitation Raman spectroscopy was integrated into the scanning electron microscope. Raman spectroscopy is not able to detect metals but adds information about elements below Beryllium, thus enables the differentiation between oxides and organic as well as inorganic material. It also provides an interpretation of the actual stoichiometry, the strain and stress, crystal symmetry, orientation, doping and crystallinity [1].

Raman spectroscopy is non-destructive and allows for the identification and quantification of solid state materials and molecules through their unique vibrational and rotational energy level structure. A laser, is focused on the sample and the scattered light is detected. In an integrated system, this is either done by means of a parabolic mirror or by moving the sample away from the electron beam to the laser beam. A small amount of the scattered light is shifted in energy from the laser frequency due to interactions with the vibrational energy levels of the molecules or the so called inelastic scattering in the solid state sample [2]. Excellent filtering is needed due to the weak Raman signal. It is essential to block the very intense laser light while still allowing high transmission of the slightly wavelength-shifted Raman scattered signal. Plotting the intensity versus frequency results in a Raman spectrum of the sample. Chemical identifications can be performed by using search algorithms against digital databases. Some typical application fields are geology, polymers, carbon materials, forensics, pharma, food industry, biomaterials and coatings.

As an example and for comparison graphene was analyzed with three different Raman spectrometers. Scanning electron microscope images revealed information about topography features like wrinkles and residues from the production process like adhesives or particles. The SEM and EDX are not able to tell whether pristine graphene or graphene oxide or reduced oxide or graphene with further functional groups was produced. A sample was first images under the electron beam and then seamlessly transferred to the Raman system (Witec, RISE) within one chamber without any doors and within the vacuum. The software allowed a point measurement and a full mapping including confocal imaging thus to generate 3D images, followed by the overlay of SEM and Raman maps. The integration of such system takes about $0,76 \mathrm{~ms}$ per spectrum. $360 \mathrm{~nm}$ is the accessible lateral resolution and a depth resolution of $1 \mu \mathrm{m}$ can be reached, depending on the used laser and objective. The laterally attached system sits at the front of the chamber by means of an extension. In the second and third experiment the Raman spectrometers (Renishaw, SCA and Clue, Horiba) were directly integrated into the SEM chamber and consisted of a retractable arm that can be moved between the pole piece and the sample. The sample was first analyzed with the electron beam, with the lowest working distance possible to achieve a high resolution image, and then moved down to approximately $16 \mathrm{~mm}$ working distance. After that, the Raman arm was inserted and a Raman measurement on exactly that point was performed. The systems allowed collocated 
SEM imaging and Raman point measurements at exactly the same area without moving the sample. The lateral resolution in this set up is approximately 2-2.5 $\mu \mathrm{m}$. Simultaneous low resolution SE and Inlens detection was possible with these set ups. The minimum resolution and scan area of all systems was basically limited by the SEM stages. Commonly three laser can easily be integrated.

\section{References:}

[1] E. Smith et al, "Modern Raman Spectroscopy A practical approach", Wiley (2013).

[2] D.J. Gardiner et al, "Practical Raman spectroscopy”, Springer, (1989).

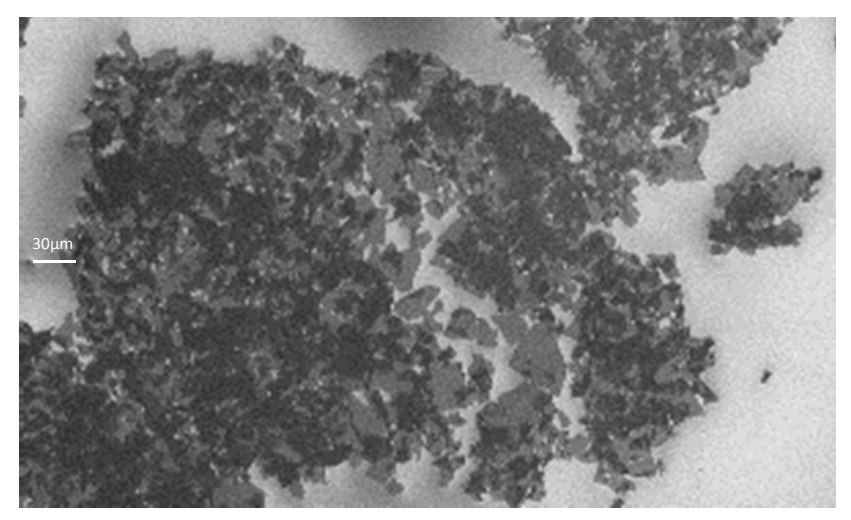

Figure 1. SEM image of graphene before using the Raman system

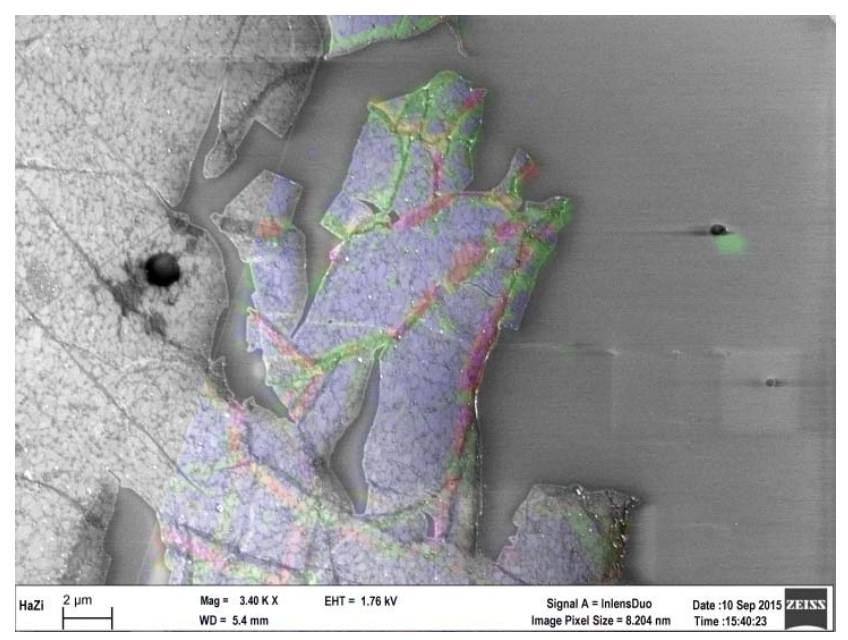

Figure 2. Overlay of SEM image and Raman mapping on CVD graphene 